\title{
Hitting times for the stochastic wave equation with fractional colored noise
}

\author{
Jorge Clarke de la Cerda and Ciprian A. Tudor
}

\begin{abstract}
We give sharp regularity results for the solution to the stochastic wave equation with linear fractional-colored noise. We apply these results in order to establish upper and lower bounds for the hitting probabilities of the solution in terms of the Hausdorff measure and the Newtonian capacity.
\end{abstract}

\section{Introduction}

The recent development of a stochastic calculus for fractional Brownian motion (fBm) naturally led to the study of stochastic partial differential equations (SPDEs) driven by this Gaussian process. The motivation comes from the wide applicability fBm. We refer to [13], [15], [17], [19] and [20] among others, for theoretical studies of SPDEs driven by fBm. To list only a few examples of the appearance of fractional noises in practical situations, we mention [14] for biophysics, [3] for financial time series, [12] for electrical engineering, and [5] for physics.

The purpose of our paper is to study the stochastic wave equation driven by fractional-colored Gaussian noise. Our work continues, in part, the line of research which concerns SPDEs driven by fBm but at the same time it follows the research line initiated by Dalang in [6] which treats equations with noise white in time and correlated in space. More precisely, we consider a system of $k$ stochastic wave equations

$$
\frac{\partial^{2} u_{i}}{\partial t^{2}}(t, x)=\Delta u_{i}(t, x)+\dot{W}_{i}(t, x), \quad t \in[0, T], x \in \mathbb{R}^{d}
$$

with initial conditions $u_{i}(t, x)=0$ and $\partial u_{i} / \partial t(0, x)=0$ for every $x \in \mathbb{R}^{d}$ and for every $i=1, \ldots, k$. The driving Gaussian process behaves as a fractional Brownian

Mathematics Subject Classification (2010): Primary 60H15; Secondary 60H05.

Keywords: Stochastic wave equation, potential theory, hitting probability, capacity, Hausdorff dimension, spatially homogeneous Gaussian noise, fractional Brownian motion, Hölder continuity. 
motion in time and has spatial covariance given by the Riesz kernel. More precisely

$$
\mathbf{E}\left(W_{i}(t, A) W_{j}(s, B)\right)=\delta_{i, j} R_{H}(t, s) \int_{A} \int_{B} f(x-y) d x d y
$$

for every $t, s \in[0, T]$ and Borel sets $A$ and $B$ in $\mathbb{R}^{d}$, where $R_{H}$ is the covariance of the fractional Brownian motion (2.4) and $f: \mathbb{R}^{d} \rightarrow \mathbb{R}_{+}$is the Fourier transform of a nonnegative tempered measure $\mu$ on $\mathbb{R}^{d}$ whose density with respect to Lebesgue measure is $|\xi|^{-(d-\beta)}, 0<\beta<d$. Above, $\delta_{i, j}$ denotes the Kronecker symbol.

The equation (1.1) has been studied recently in [2] for $H>1 / 2$. It has been proven that (1.1) admits a unique mild solution if and only if $\beta<2 H+1$, this extends the result obtained in [6] in the case $H=1 / 2$. The purpose of the present paper is to analyze further the solution of (1.1). We will actually give sharp results for its regularity, in time and in space, and we apply these regularity results to study the hitting probabilities for the solution $u$ of (1.1). More precisely, given a Borel set $A \subset \mathbb{R}^{k}$ we want to determine whether the process $\left(u(t, x), t \in[0, T], x \in \mathbb{R}^{d}\right)$ hits the set $A$ with positive probability. Recently, there have been several papers on hitting probabilities, and more generally speaking, on potential theory for systems of SPDEs. We refer, among others, to [7], [8], [9], [10], and [16]. The study of hitting probabilities for stochastic partial differential equations with fractional noise in time is new. As far as we know, only the paper [18] treats this problem. Actually, in this reference the authors give upper and lower bounds for the hitting times of solutions to a system of stochastic heat equations on the circle with noise fractional in time.

Our aim is to advance this research direction. As we mentioned before, we make a potential analysis of the solution to the stochastic wave equation with fractional colored noise. That means the noise behaves as fractional Brownian motion with respect to the time variable and it has "colored"non-white spatial covariance. In our work this spatial covariance will be described by the Riesz kernel. It is now widely accepted that in order to obtain results on the hitting times of a stochastic process, a detailed analysis of the behavior of the increments of the process is needed. We address this question and we find the following: the solution $u(t, x), t \in[0, T], x \in \mathbb{R}^{d}$ to (1.1) is Hölder continuous of order $\frac{1}{2}(2 H+1-\beta), \beta \in$ $(2 H-1, d \wedge 2 H+1)$, in time as well as in space. This generalizes the result obtained in [10] and [11] for the wave equation with white noise in time and Riesz covariance in space. Although the main lines of our work follow the approach of [11], we stress that, as usual, the fractional case involves more complex calculation and the techniques used in the standard white noise case need to be adapted. This is mainly due to the nature of the noise and to the structure of the Gaussian space associated to the noise. We will point out later how the fractional noise requires a more complex analysis than in e.g. [8] or [11]. Moreover, the study of the solution to the wave equation is generally recognized to be more difficult than, for example, the solution to the heat equation, due to the appearance of the trigonometric functions, and this is also the case in our work.

We mention that there are more or less general criteria for determining the hitting times for a stochastic process. Such criteria have been given in [4], [8], [9], 
and [11] among others. We will use the approach in [4] because it concerns Gaussian processes and fits our context well (note that the solution to (1.1) is Gaussian).

Our paper is structured as follows. Section 2 contains some preliminaries, briefly describe the basic properties of the Gaussian noise and its associated Hilbert space, we list the elements of the potential theory that we will use and we recall some facts related of the solution to the stochastic wave equation with fractionalcolored noise. In Section 3 we analyze the Hölder regularity of the solution with respect to its time and space variables. Section 4 is devoted to the study of the hitting probabilities for this solution, based on a criterion in [4].

\section{Preliminaries}

This section introduce the basic notions that we will need throughout the paper. We first introduce the canonical Hilbert space associated to the fractional-colored Gaussian noise. In part 2.2 we present the basics of the potential theory that is needed.

\subsection{The canonical Hilbert space}

We denote by $C_{0}^{\infty}\left(\mathbb{R}^{d+1}\right)$ the space of infinitely differentiable functions on $\mathbb{R}^{d+1}$ with compact support, and by $\mathcal{S}\left(\mathbb{R}^{d}\right)$ the Schwartz space of rapidly decreasing $C^{\infty}$ functions in $\mathbb{R}^{d}$. For $\varphi \in L^{1}\left(\mathbb{R}^{d}\right)$, we let $\mathcal{F} \varphi$ be the Fourier transform of $\varphi$ :

$$
\mathcal{F} \varphi(\xi)=\int_{\mathbb{R}^{d}} e^{-i \xi \cdot x} \varphi(x) d x
$$

We begin by introducing the framework of [6]. Let $\mu$ be a nonnegative tempered measure on $\mathbb{R}^{d}$, i.e., a nonnegative measure which satisfies

$$
\int_{\mathbb{R}^{d}}\left(\frac{1}{1+|\xi|^{2}}\right)^{l} \mu(d \xi)<\infty, \quad \text { for some } l>0 .
$$

Since the integrand is nonincreasing in $l$, we may assume that $l \geq 1$ is an integer. Note that $1+|\xi|^{2}$ behaves as a constant near 0 , and as $|\xi|^{2}$ at $\infty$, and hence $(2.1)$ is equivalent to:

$$
\int_{|\xi| \leq 1} \mu(d \xi)<\infty, \quad \text { and } \quad \int_{|\xi| \geq 1} \mu(d \xi) \frac{1}{|\xi|^{2 l}}<\infty, \quad \text { for some integer } l \geq 1
$$

Let $f: \mathbb{R}^{d} \rightarrow \mathbb{R}_{+}$be the Fourier transform of $\mu$ in $\mathcal{S}^{\prime}\left(\mathbb{R}^{d}\right)$, i.e.,

$$
\int_{\mathbb{R}^{d}} f(x) \varphi(x) d x=\int_{\mathbb{R}^{d}} \mathcal{F} \varphi(\xi) \mu(d \xi), \quad \forall \varphi \in \mathcal{S}\left(\mathbb{R}^{d}\right) .
$$

Simple properties of the Fourier transform show that, for any $\varphi, \psi \in \mathcal{S}\left(\mathbb{R}^{d}\right)$,

$$
\int_{\mathbb{R}^{d}} \int_{\mathbb{R}^{d}} \varphi(x) f(x-y) \psi(y) d x d y=\int_{\mathbb{R}^{d}} \mathcal{F} \varphi(\xi) \overline{\mathcal{F} \psi(\xi)} \mu(d \xi) .
$$


An approximation argument shows that the previous equality also holds for indicator functions $\varphi=1_{A}$ and $\psi=1_{B}$, with $A$ and $B \in \mathcal{B}_{b}\left(\mathbb{R}^{d}\right)$, where $\mathcal{B}_{b}\left(\mathbb{R}^{d}\right)$ is the class of bounded Borel sets of $\mathbb{R}^{d}$ :

$$
\int_{A} \int_{B} f(x-y) d x d y=\int_{\mathbb{R}^{d}} \mathcal{F} 1_{A}(\xi) \overline{\mathcal{F} 1_{B}(\xi)} \mu(d \xi) .
$$

Now we introduce the fractional Brownian motion ( $\mathrm{fBm}$ ) with Hurst index $H \in(0,1)$. This is a mean zero Gaussian process $\left(B_{t}^{H}\right)_{t \in[0, T]}$ with covariance

$$
R_{H}(t, s):=\frac{1}{2}\left(t^{2 H}+s^{2 H}-|t-s|^{2 H}\right), \quad t, s \in[0, T] .
$$

Let us denote by $\mathcal{H}$ the canonical Hilbert space associated with this Gaussian process. This Hilbert space is defined as the closure of the linear space generated by the indicator functions $1_{[0, t]}, t \in[0, T]$, with respect to the inner product

$$
\left\langle 1_{[0, t]}, 1_{[0, s]}\right\rangle_{\mathcal{H}}=R_{H}(t, s) \text {. }
$$

It is well known that for $H>1 / 2$ we have the expression

$$
R_{H}(t, s)=\alpha_{H} \int_{0}^{t} \int_{0}^{s}|u-v|^{2 H-2} d u d v
$$

for every $s, t \in[0, T]$ with $\alpha_{H}:=H(2 H-1)$. More generally, for $H>1 / 2$ and every $\psi, \phi \in \mathcal{H}=\mathcal{H}([0, T])$ we have

$$
\langle\psi, \phi\rangle_{\mathcal{H}}=\alpha_{H} \int_{0}^{T} \int_{0}^{T} \psi(u) \phi(v)|u-v|^{2 H-2} d u d v
$$

As in [1], on a complete probability space $(\Omega, \mathcal{F}, P)$, we consider a zero-mean Gaussian process $W=\left\{W_{t}(A) ; t \geq 0, A \in \mathcal{B}_{b}\left(\mathbb{R}^{d}\right)\right\}$ with covariance

$$
\mathbf{E}\left(W_{t}(A) W_{s}(B)\right)=R_{H}(t, s) \int_{A} \int_{B} f(x-y) d x d y=:\left\langle 1_{[0, t] \times A}, 1_{[0, s] \times B}\right\rangle_{\mathcal{H P}}
$$

Let $\mathcal{E}$ be the set of linear combinations of elementary functions $1_{[0, t] \times A}, t \geq 0$, let $A \in \mathcal{B}_{b}\left(\mathbb{R}^{d}\right)$, and let $\mathcal{H} \mathcal{P}$ be the Hilbert space defined as the closure of $\mathcal{E}$ with respect to the inner product $\langle\cdot, \cdot\rangle_{\mathcal{H P}}$. (Alternatively, $\mathcal{H P}$ can be defined as the completion of $C_{0}^{\infty}\left(\mathbb{R}^{d+1}\right)$ with respect to the inner product $\langle\cdot, \cdot\rangle_{\mathcal{H} \mathcal{P}}$; see [1].)

The map $1_{[0, t] \times A} \mapsto W_{t}(A)$ is an isometry between $\mathcal{E}$ and the Gaussian space $H^{W}$ of $W$, which can be extended to $\mathcal{H} \mathcal{P}$. We denote this extension by

$$
\varphi \mapsto W(\varphi)=\int_{0}^{\infty} \int_{\mathbb{R}^{d}} \varphi(t, x) W(d t, d x) .
$$

In the present paper, we assume that $H>1 / 2$. Hence, (2.5) holds. From (2.3) and $(2.5)$, it follows that for any $\varphi, \psi \in \mathcal{E}$,

$$
\begin{aligned}
\langle\varphi, \psi\rangle_{\mathcal{H P}} & =\alpha_{H} \int_{0}^{\infty} \int_{0}^{\infty} \int_{\mathbb{R}^{d}} \int_{\mathbb{R}^{d}} \varphi(u, x) \psi(v, y) f(x-y)|u-v|^{2 H-2} d x d y d u d v \\
& =\alpha_{H} \int_{0}^{\infty} \int_{0}^{\infty} \int_{\mathbb{R}^{d}} \mathcal{F} \varphi(u, \cdot)(\xi) \overline{\mathcal{F} \psi(v, \cdot)(\xi)}|u-v|^{2 H-2} \mu(d \xi) d u d v .
\end{aligned}
$$


Moreover, we can interchange the order of the integrals $d u d v$ and $\mu(d \xi)$, since for indicator functions $\varphi$ and $\psi$, the integrand is a product of a function of $(u, v)$ and a function of $\xi$. Hence, for $\varphi, \psi \in \mathcal{E}$, we have

$$
\langle\varphi, \psi\rangle_{\mathcal{H P}}=\alpha_{H} \int_{\mathbb{R}^{d}} \int_{0}^{\infty} \int_{0}^{\infty} \mathcal{F} \varphi(u, \cdot)(\xi) \overline{\mathcal{F} \psi(v, \cdot)(\xi)}|u-v|^{2 H-2} d u d v \mu(d \xi) .
$$

The space $\mathcal{H} \mathcal{P}$ may contain distributions, but it contains the space $|\mathcal{H P}|$ of measurable functions $\varphi: \mathbb{R}_{+} \times \mathbb{R}^{d} \rightarrow \mathbb{R}$ such that

$$
\|\varphi\|_{|\mathcal{H P}|}^{2}:=\alpha_{H} \int_{0}^{\infty} \int_{0}^{\infty} \int_{\mathbb{R}^{d}} \int_{\mathbb{R}^{d}}|\varphi(u, x)||\varphi(v, y)| f(x-y)|u-v|^{2 H-2} d x d y d u d v<\infty .
$$

\subsection{Elements of the potential theory}

Our aim is to analyze the probability

$$
P(u(I) \cap A) \neq \emptyset
$$

where $u$ is the solution to (1.1), $I$ is a Borel set included in $[0, T] \times \mathbb{R}^{d}$, and $A$ is a Borel set in $\mathbb{R}^{k}$. Here $u(I)$ means the image of $I$ under the random map $(t, x) \rightarrow u(t, x)$.

We will briefly describe the notions from potential theory that we will need. For all Borel sets $F \subset \mathbb{R}^{d}$ we define $\mathcal{P}(F)$ to be the set of all probability measures with compact support included in $F$. For all $\mu \in \mathcal{P}\left(\mathbb{R}^{d}\right)$, we denote by $I_{\beta}(\mu)$ the so-called $\beta$-energy defined by

$$
I_{\beta}(\mu)=\int_{\mathbb{R}^{d}} \int_{\mathbb{R}^{d}} K_{\beta}(\|x-y\|) \mu(d x) \mu(d y)
$$

where

$$
K_{\beta}(r)= \begin{cases}r^{-\beta} & \text { if } \beta>0 \\ \log \left(N_{0} / r\right) & \text { if } \beta=0 \\ 1 & \text { if } \beta<0\end{cases}
$$

Here $N_{0}$ is a constant.

For all $\beta \in \mathbb{R}$ and $F \in \mathcal{B}\left(\mathbb{R}^{d}\right)$ we define the $\beta$-dimensional capacity of $F$ by

$$
\operatorname{Cap}_{\beta}(F)=\left[\inf _{\mu \in \mathcal{P}(F)} I_{\beta}(\mu)\right]^{-1}
$$

with the convention $1 / \infty:=0$. The $\beta$-dimensional Hausdorff measure of the set $F \in \mathcal{B}\left(\mathbb{R}^{d}\right)$ is given by

$$
\mathcal{H}_{\beta}(F)=\lim _{\varepsilon \rightarrow 0^{+}} \inf \left[\sum_{i=1}^{\infty}\left(2 r_{i}\right)^{\beta} ; F \subset \bigcup_{i=1}^{\infty} B\left(x_{i}, r_{i}\right), \sup _{i \geq 1} r_{i} \leq \varepsilon\right]
$$

where $B(x, r)$ denotes the Euclidean ball of radius $r>0$ centered at $x \in \mathbb{R}^{d}$. When $\beta<0$, the $\beta$-dimensional Hausdorff measure of $F$ is infinite by definition. 


\subsection{The stochastic wave equation with linear fractional-colored noise}

Consider the linear stochastic wave equation driven by an infinite-dimensional fractional Brownian motion $W$ with Hurst parameter $H \in(0,1)$. That is

$$
\left\{\begin{aligned}
\frac{\partial^{2} u}{\partial t^{2}}(t, x) & =\Delta u(t, x)+\dot{W}(t, x), \quad t>0, x \in \mathbb{R}^{d} \\
u(0, x) & =0, \quad x \in \mathbb{R}^{d} \\
\frac{\partial u}{\partial t}(0, x) & =0, \quad x \in \mathbb{R}^{d}
\end{aligned}\right.
$$

Here $\Delta$ is the Laplacian on $\mathbb{R}^{d}$ and $W=\left\{W_{t}(A) ; t \geq 0, A \in \mathcal{B}_{b}\left(\mathbb{R}^{d}\right)\right\}$ is a centered Gaussian field with covariance

$$
\mathbf{E}\left(W_{t}(A) W_{s}(B)\right)=R_{H}(t, s) \int_{A} \int_{B} f(x-y) d x d y,
$$

where $R_{H}$ is the covariance of the fractional Brownian motion and $f$ is the Riesz kernel.

Let $G_{1}$ be the fundamental solution of $u_{t t}-\Delta u=0$. It is known that $G_{1}(t, \cdot)$ is a distribution in $\mathcal{S}^{\prime}\left(\mathbb{R}^{d}\right)$ with rapid decrease, and

$$
\mathcal{F} G_{1}(t, \cdot)(\xi)=\frac{\sin (t|\xi|)}{|\xi|},
$$

for any $\xi \in \mathbb{R}^{d}, t>0, d \geq 1$ (see e.g. [21]). In particular,

$$
\begin{array}{ll}
G_{1}(t, x)=\frac{1}{2} 1_{\{|x|<t\}}, & \text { if } d=1, \\
G_{1}(t, x)=\frac{1}{2 \pi} \frac{1}{\sqrt{t^{2}-|x|^{2}}} 1_{\{|x|<t\}}, & \text { if } d=2, \\
G_{1}(t, x)=c_{d} \frac{1}{t} \sigma_{t}, & \text { if } d=3,
\end{array}
$$

where $\sigma_{t}$ denotes the surface measure on the 3 -dimensional sphere of radius $t$.

The solution of (2.13) is a square-integrable process $u=\left\{u(t, x) ; t \geq 0, x \in \mathbb{R}^{d}\right\}$ defined by

$$
u(t, x)=\int_{0}^{t} \int_{\mathbb{R}^{d}} G_{1}(t-s, x-y) W(d s, d y) .
$$

By definition, $u(t, x)$ exists if and only if the stochastic integral above is well defined, i.e., $g_{t x}:=G_{1}(t-\cdot, x-\cdot) \in \mathcal{H} \mathcal{P}$. In this case, $\mathbf{E}|u(t, x)|^{2}=\left\|g_{t x}\right\|_{\mathcal{H} \mathcal{P}}^{2}$.

The following result was proved in [2].

Theorem 2.1. The stochastic wave equation (2.13) admits a unique mild solution $(u(t, x))_{t \in[0, T], x \in \mathbb{R}^{d}}$ if and only if

$$
\int_{\mathbb{R}^{d}}\left(\frac{1}{1+|\xi|^{2}}\right)^{H+1 / 2} \mu(d \xi)<\infty .
$$


Remark 2.2. Note that (2.16) is equivalent to

$$
\int_{|\xi| \leq 1} \mu(d \xi)<\infty, \quad \text { and } \quad \int_{|\xi| \geq 1} \mu(d \xi) \frac{1}{|\xi|^{2 H+1}}<\infty .
$$

As mentioned in the introduction, we will consider throughout the paper that the spatial covariance of the noise $W$ is given by the Riesz kernel. This means the measure $\mu$ is

$$
d \mu(\xi)=|\xi|^{-d+\beta} d \xi \quad \text { with } \beta \in(0, d)
$$

In this case the kernel $f$ is given by

$$
f(\xi)=|\xi|^{-\beta} \quad \text { with } \beta \in(0, d) .
$$

Note that in the case of the Riesz kernel, condition (2.16) is equivalent to

$$
\beta \in(0, d \wedge(2 H+1)) .
$$

Remark 2.3. Since $H>1 / 2$ and so $2 H+1 \in(2,3)$, for dimension $d=1,2$ we have $\beta \in(0, d)$ while for $d \geq 3$ we have $\beta \in(0,2 H+1)$.

\section{Regularity of the solution}

\subsection{Regularity in time}

In this part we focus our attention on the behavior of the increments of the solution $u(t, x)$ with respect to the variable $t$. We give upper and lower bounds for the $L^{2}$-norm of this increment. Usually, obtaining upper bounds is considered easier than obtaining lower bounds. This is the case in our work. Actually, to obtain the sharp regularity of $u$ with respect to the time variable, we need to impose a stronger assumption than (2.18) on the parameters $\beta$ and $H$ (condition (3.1) below). This is due to the characteristics of the scalar product in $\mathcal{H P}$.

We will start with the following useful lemma that gives an explicit expression for the $\mathcal{H}$ norm of the cosine and sine functions.

Lemma 3.1. Let $f(x)=\cos (x)$ and $g(x)=\sin x$ for $x \in \mathbb{R}$. Then, for every $a, b \in \mathbb{R}, a<b$,

$$
\begin{aligned}
\left\|f 1_{(a, b)}\right\|_{\mathcal{H}}^{2}= & \alpha_{H} \int_{0}^{b-a} d v \cos (v) v^{2 H-2}(b-a-v) \\
& +\alpha_{H} \cos (a+b) \int_{0}^{b-a} d v v^{2 H-2} \sin (b-a-v) .
\end{aligned}
$$

and

$$
\begin{aligned}
\left\|g 1_{(a, b)}\right\|_{\mathcal{H}}^{2}= & \alpha_{H} \int_{0}^{b-a} d v \cos (v) v^{2 H-2}(b-a-v) \\
& -\alpha_{H} \cos (a+b) \int_{0}^{b-a} d v v^{2 H-2} \sin (b-a-v) .
\end{aligned}
$$


Proof. Using the expression of the scalar product in the Hilbert space $\mathcal{H}$ and the trigonometric identities we can write

$$
\begin{aligned}
\left\|f 1_{(a, b)}\right\|_{\mathcal{H}}^{2}+\left\|g 1_{(a, b)}\right\|_{\mathcal{H}}^{2} & =\alpha_{H} \int_{a}^{b} \int_{a}^{b}|u-v|^{2 H-2}(\cos u \cos v+\sin u \sin v) d u d v \\
& =\alpha_{H} \int_{a}^{b} d u \int_{a}^{b} d v|u-v|^{2 H-2} \cos (u-v) \\
& =2 \alpha_{H} \int_{a}^{b} d u \int_{0}^{u-a} d v \cos (v) v^{2 H-2} \\
& =2 \alpha_{H} \int_{0}^{b-a} d v \cos (v) v^{2 H-2}(b-a-v)
\end{aligned}
$$

where we made the change of variables $\tilde{v}=u-v$ in the integral with respect to $v$ and we computed the integral with respect to $u$. Similarly

$$
\begin{aligned}
\left\|f 1_{(a, b)}\right\|_{\mathcal{H}}^{2}-\left\|g 1_{(a, b)}\right\|_{\mathcal{H}}^{2} & =\alpha_{H} \int_{a}^{b} d u \int_{a}^{b} d v|u-v|^{2 H-2}(\cos u \cos v-\sin u \sin v) \\
& =\alpha_{H} \int_{a}^{b} \int_{a}^{b}|u-v|^{2 H-2} \cos (u+v) d u d v
\end{aligned}
$$

and by the change of variable $\tilde{v}=u-v$ in the integral with respect to $v$,

$$
\begin{aligned}
\left\|f 1_{(a, b)}\right\|_{\mathcal{H}}^{2}-\left\|g 1_{(a, b)}\right\|_{\mathcal{H}}^{2} & =2 \alpha_{H} \int_{a}^{b} d u \int_{0}^{u-a} d v \cos (2 u-v) v^{2 H-2} \\
& =2 \alpha_{H} \int_{0}^{b-a} d v v^{2 H-2} \int_{v+a}^{b} d u \cos (2 u-v) \\
& =\alpha_{H} \int_{0}^{b-a} d v v^{2 H-2}(\sin (2 b-v)-\sin (2 a+v)) \\
& =2 \alpha_{H} \cos (a+b) \int_{0}^{b-a} d v v^{2 H-2} \sin (b-a-v) .
\end{aligned}
$$

Remark 3.2. As a consequence of Lemma 3.1 we deduce the following:

(i) For every $a, b \in \mathbb{R}, a<b$,

$$
\left\|f 1_{(a, b)}\right\|_{\mathcal{H}}^{2} \leq 2 \alpha_{H} \int_{0}^{b-a} d v \cos (v) v^{2 H-2}(b-a-v) .
$$

(ii) For any $x>0$ the quantity $\int_{0}^{x} v^{2 H-2} \cos (v)(x-v) d v$ is positive (it is the sum of two norms).

(iii) For every $a, b \in \mathbb{R}, a<b$,

$$
\left\|f 1_{(a, b)}\right\|_{\mathcal{H}}^{2} \geq 2 \alpha_{H} \cos (a+b) \int_{0}^{b-a} d v v^{2 H-2} \sin (b-a-v) .
$$


Later, we use also the following lemma.

Lemma 3.3. For every $a, b \in \mathbb{R}$ with $a<b$,

$$
\int_{a}^{b} \int_{a}^{b} d u d v \sin (u-v)|u-v|^{2 H-2}=0 .
$$

Proof. This follows from the trivial equality

$$
\int_{a}^{b} \int_{a}^{b} \sin (u) \cos (v)|u-v|^{2 H-2} d u d v=\int_{a}^{b} \int_{a}^{b} \sin (v) \cos (u)|u-v|^{2 H-2} d u d v
$$

Concretely, we will prove the following result concerning regularity in time of the solution to (2.13). We mention that, in the rest of our paper, $c, C$, etc. will denote generic positive constants that may change from line to line.

Proposition 3.4. Assume that

$$
\beta \in(2 H-1, d \wedge(2 H+1)) .
$$

Let $t_{0}, M>0$ and fix $x \in[-M, M]^{d}$. Then there exist positive constants $c_{1}$ and $c_{2}$ such that, for every $s, t \in\left[t_{0}, T\right]$,

$$
c_{1}|t-s|^{2 H+1-\beta} \leq \mathbf{E}|u(t, x)-u(s, x)|^{2} \leq c_{2}|t-s|^{2 H+1-\beta} .
$$

Proof. We let $h>0$ and we estimate the $L^{2}(\Omega)$-norm of the increment $u(t+h, x)-$ $u(t, x)$. Splitting the interval $[0, t+h]$ into the intervals $[0, t]$ and $[t, t+h]$, and using the inequality $|a+b|^{2} \leq 2\left(a^{2}+b^{2}\right)$, we obtain

$$
\begin{aligned}
\mathbf{E}|u(t+h, x)-u(t, x)|^{2} & \leq 2\left\{\left\|\left(g_{t+h, x}-g_{t, x}\right) 1_{[0, t]}\right\|_{\mathcal{H \mathcal { P }}}^{2}+\left\|g_{t+h, x} 1_{[t, t+h]}\right\|_{\mathcal{H \mathcal { P }}}^{2}\right\} \\
& =: 2\left[E_{1, t}(h)+E_{2}(h)\right] .
\end{aligned}
$$

The first summand can be evaluated as

$$
\begin{aligned}
& E_{1, t}(h)=\alpha_{H} \int_{\mathbb{R}^{d}} \mu(d \xi) \int_{0}^{t} \int_{0}^{t} d v d v|u-v|^{2 H-2} \mathcal{F}\left(g_{t+h, x}-g_{t x}\right)(u, \cdot)(\xi) \\
& \times \overline{\mathcal{F}\left(g_{t+h, x}-g_{t x}\right)(v, \cdot)(\xi)} \\
& =\alpha_{H} \int_{\mathbb{R}^{d}} \mu(d \xi) \int_{0}^{t} \int_{0}^{t} d u d v|u-v|^{2 H-2}\left[\mathcal{F} G_{1}(u+h, \cdot)(\xi)-\mathcal{F} G_{1}(u, \cdot)(\xi)\right] \\
& \times \overline{\mathcal{F} G_{1}(v+h, \cdot)(\xi)-\mathcal{F} G_{1}(v, \cdot)(\xi)} \\
& =\alpha_{H} \int_{0}^{t} \int_{0}^{t} d u d v|u-v|^{2 H-2} I_{h} \text {, }
\end{aligned}
$$

where

$$
\begin{aligned}
I_{h}=\int_{\mathbb{R}^{d}} \mu(d \xi)\left[\mathcal{F} G_{1}(u+h, \cdot)(\xi)-\mathcal{F} G_{1}(u, \cdot)(\xi)\right] \\
\quad \times\left[\overline{\mathcal{F} G_{1}(v+h, \cdot)(\xi)-\mathcal{F} G_{1}(v, \cdot)(\xi)}\right] \\
=\int_{\mathbb{R}^{d}} \mu(d \xi) \frac{(\sin ((u+h)|\xi|)-\sin (u|\xi|))}{|\xi|} \frac{(\sin ((v+h)|\xi|)-\sin (v|\xi|))}{|\xi|} .
\end{aligned}
$$


Using trigonometric identities we obtain

$$
\begin{aligned}
E_{1, t}(h)=\alpha_{H} \int_{0}^{t} & \int_{0}^{t} d u d v|u-v|^{2 H-2} \\
& \times \int_{\mathbb{R}^{d}} \mu(d \xi) \frac{\sin \left(\frac{h|\xi|}{2}\right)^{2}}{|\xi|^{2}} \cos \left(\frac{(2 u+h)|\xi|}{2}\right) \cos \left(\frac{(2 v+h)|\xi|}{2}\right) \\
=c \cdot \alpha_{H} & \int_{0}^{t} \int_{0}^{t} d u d v|u-v|^{2 H-2} \\
& \times \int_{\mathbb{R}^{d}} \frac{d \xi}{|\xi|^{d-\beta+2}} \sin (h|\xi|)^{2} \cos ((2 u+h)|\xi|) \cos ((2 v+h)|\xi|) .
\end{aligned}
$$

Making the change of variables $\tilde{u}=(2 u+h)|\xi|$ and $\tilde{v}=(2 v+h)|\xi|$, we obtain

$$
\begin{aligned}
E_{1, t}(h)=c \cdot \alpha_{H} & \int_{\mathbb{R}^{d}} \frac{d \xi}{|\xi|^{d-\beta+2 H+2}} \sin (h|\xi|)^{2} \\
& \times \int_{h|\xi|}^{(2 t+h)|\xi|} \int_{h|\xi|}^{(2 t+h)|\xi|} d u d v|u-v|^{2 H-2} \cos u \cos v \\
=c & \int_{\mathbb{R}^{d}} \frac{d \xi}{|\xi|^{d-\beta+2 H+2}} \sin (h|\xi|)^{2}\left\|\cos (\cdot) 1_{(h|\xi|,(2 t+h)|\xi|)}(\cdot)\right\|_{\mathcal{H}}^{2}
\end{aligned}
$$

and using Lemma 3.1,

$$
\begin{aligned}
E_{1, t}(h)= & c \cdot \alpha_{H} \int_{\mathbb{R}^{d}} \frac{d \xi}{|\xi|^{d-\beta+2 H+2}} \sin (h|\xi|)^{2} \\
\times & \times\left[\int_{0}^{2 t|\xi|} \cos (v) v^{2 H-2}(2 t|\xi|-v) d v\right. \\
& \left.\quad+\cos (2 t|\xi|+2 h|\xi|) \int_{0}^{2 t|\xi|} v^{2 H-2}(\sin (2 t|\xi|-v))\right] \\
= & c \cdot \alpha_{H} \int_{\mathbb{R}^{d}} \frac{d \xi}{|\xi|^{d-\beta+2 H+2}} \sin (h|\xi|)^{2} \times\left[2 t|\xi| \int_{0}^{2 t|\xi|} \cos (v) v^{2 H-2} d v\right. \\
& -\sin (2 t|\xi|)(2 t|\xi|)^{2 H-1}+(2 H-1) \int_{0}^{2 t|\xi|} \sin (v) v^{2 H-2} d v \\
& \left.+\cos (2 t|\xi|+2 h|\xi|) \int_{0}^{2 t|\xi|} v^{2 H-2}(\sin (2 t|\xi|-v))\right]
\end{aligned}
$$

where we use integration by parts. By point (i) of Remark 3.2, we have the upper bound

$$
\begin{aligned}
E_{1, t}(h) \leq & c \cdot \alpha_{H} \int_{\mathbb{R}^{d}} \frac{d \xi}{|\xi|^{d-\beta+2 H+2}} \sin (h|\xi|)^{2} \\
\times & \times\left[2 t|\xi| \int_{0}^{2 t|\xi|} \cos (v) v^{2 H-2} d v-\sin (2 t|\xi|)(2 t|\xi|)^{2 H-1}\right. \\
& \left.\quad+(2 H-1) \int_{0}^{2 t|\xi|} \sin (v) v^{2 H-2} d v\right]
\end{aligned}
$$


We will treat the three summands above separately. For the first,

$$
\begin{aligned}
\int_{\mathbb{R}^{d}} \frac{d \xi}{|\xi|^{d-\beta+2 H+2}} \sin (h|\xi|)^{2} 2 t|\xi| \int_{0}^{2 t|\xi|} \cos (v) v^{2 H-2} d v \\
\quad=c_{t, H} h^{2 H+1-\beta}\left|\int_{\mathbb{R}^{d}} \frac{d \xi}{|\xi|^{d-\beta+2 H+1}} \sin (|\xi|)^{2} \int_{0}^{\frac{2 t|\xi|}{h}} \cos (v) v^{2 H-2} d v\right| \\
\leq c_{t, H} h^{2 H+1-\beta} \int_{\mathbb{R}^{d}} \frac{d \xi}{|\xi|^{d-\beta+2 H+1}} \sin (|\xi|)^{2}\left|\int_{0}^{\frac{2 t|\xi|}{h}} \cos (v) v^{2 H-2} d v\right| \\
\leq c_{t, H} h^{2 H+1-\beta}
\end{aligned}
$$

where we have used condition (2.18) and the fact that the integral

$$
\int_{0}^{\infty} \cos (v) v^{2 H-2} d v
$$

is convergent (this implies that the function $x \in[0, \infty) \rightarrow \int_{0}^{x} \cos (v) v^{2 H-2} d v$ admits a limit at infinity and is therefore bounded). On the other hand,

$$
\begin{aligned}
\int_{\mathbb{R}^{d}} \frac{d \xi}{|\xi|^{d-\beta+2 H+2}} & \sin (h|\xi|)^{2} \sin (2 t|\xi|)(2 t|\xi|)^{2 H-1} \\
= & c_{t} h^{3-\beta} \int_{\mathbb{R}^{d}} \frac{d \xi}{|\xi|^{d-\beta+3}} \sin (|\xi|)^{2} \sin \left(\frac{2 t|\xi|}{h}\right) \\
= & c_{t} h^{3-\beta} \int_{|\xi| \leq 1} \frac{d \xi}{|\xi|^{d-\beta+3}} \sin (|\xi|)^{2} \sin \left(\frac{2 t|\xi|}{h}\right) \\
& +c_{t} h^{3-\beta} \int_{|\xi|>1} \frac{d \xi}{|\xi|^{d-\beta+3}} \sin (|\xi|)^{2} \sin \left(\frac{2 t|\xi|}{h}\right) .
\end{aligned}
$$

The second part over the region $|\xi| \geq 1$ is bounded by $c h^{3-\beta}$ simply because the sine is bounded from above by 1 . The second integral has a singularity for $|\xi|$ close to zero. Using that $\sin (x) \leq x$ for all $x \geq 0$, we will bound it above by

$$
\begin{aligned}
h^{3-\beta} \int_{|\xi| \leq 1} & \frac{d \xi}{|\xi|^{d-\beta+3}} \sin (|\xi|)^{2} \sin \left(\frac{2 t|\xi|}{h}\right) \\
& \leq c_{t} h^{3-\beta} \int_{|\xi| \leq 1} \frac{d \xi}{|\xi|^{d-\beta+3}}|\xi|^{2}\left|\sin \left(\frac{2 t|\xi|}{h}\right)\right|^{2-2 H}\left|\sin \left(\frac{2 t|\xi|}{h}\right)\right|^{2 H-1} \\
& \leq c_{t} h^{2 H+1-\beta} \int_{|\xi| \leq 1} \frac{d \xi}{|\xi|^{d-\beta+2 H-1}}
\end{aligned}
$$

where we have bounded $|\sin (2 t|\xi| / h)|^{2-2 H}$ by $c_{t}\left(|\xi| h^{-1}\right)^{2-2 H}$ and $|\sin (2 t|\xi| / h)|^{2 H-1}$ by 1 . The last integral is finite since $\beta>2 H-1$ (assumption (3.1)). 
Finally

$$
\begin{aligned}
\int_{\mathbb{R}^{d}} \frac{d \xi}{|\xi|^{d-\beta+2 H+2}} \sin (h|\xi|)^{2} \int_{0}^{2 t|\xi|} \sin (v) v^{2 H-2} d v & \sin (|\xi|)^{2} \int_{0}^{2 t|\xi| / h} \sin (v) v^{2 H-2} d v \\
= & h^{2 H+2-\beta} \int_{\mathbb{R}^{d}} \frac{d \xi}{|\xi|^{d-\beta+2 H+2}} \sin (|\xi|)^{2} \int_{0}^{2 t|\xi| / h} \sin (v) v^{2 H-2} d v \\
= & h^{2 H+2-\beta} \int_{|\xi| \leq 1} \frac{d \xi}{|\xi|^{d-\beta+2 H+2}} \sin (|\xi|)^{2} \int_{0}^{2 t|\xi| / h} \sin (v) v^{2 H-2} d v \\
& +h^{2 H+2-\beta} \int_{|\xi| \geq 1} \frac{d \xi}{|\xi|^{d-\beta+2 H+2}} \sin \int_{|\xi| \leq 1}^{2 t|\xi| / h} \frac{d \xi}{|\xi|^{d-\beta+2 H+2}}|\sin v| v^{2 H-2} d v \\
\leq & h^{2 H+2-\beta} \int_{|\xi| \geq 1} \frac{d \xi}{|\xi|^{d-\beta+2 H+2}} \int_{0}^{2 t|\xi| / h} \sin (v) v^{2 H-2} d v .
\end{aligned}
$$

Using again the fact that

$$
\int_{0}^{\infty} \sin (v) v^{2 H-2} d v
$$

is convergent it is easy to see that the integral over the region $|\xi| \geq 1$ is bounded by $c_{t} h^{2 H+2-\beta}$. For the integral over $|\xi| \leq 1$ we make the change of variables $\tilde{v}=v h / \xi$ and we get

$$
\begin{aligned}
& h^{3-\beta} \int_{|\xi| \leq 1} \frac{d \xi}{|\xi|^{d-\beta+1}} \int_{0}^{2 t}\left|\sin \left(\frac{v|\xi|}{h}\right)\right| v^{2 H-2} d v \\
& \quad=h^{3-\beta} \int_{|\xi| \leq 1} \frac{d \xi}{|\xi|^{d-\beta+1}} \int_{0}^{2 t}\left|\sin \left(\frac{v|\xi|}{h}\right)\right|^{2-2 H}\left|\sin \left(\frac{v|\xi|}{h}\right)\right|^{2 H-1} v^{2 H-2} d v \\
& \quad \leq c_{t} h^{2 h+1-\beta} \int_{|\xi| \leq 1} \frac{d \xi}{|\xi|^{d-\beta+2 H-1}},
\end{aligned}
$$

where we have made the same analysis as for the second summand in the decomposition of $E_{1, t}(h)$. In this way, we obtained the upper bound

$$
E_{1, t}(h) \leq C h^{2 H+1-\beta},
$$

for the summand $E_{1, t}(h)$ in $(3.2)$.

Now we study the term $E_{2}(h)$ in $(3.2)$ (the notation $E_{2}(h)$ instead of $E_{2, t}(h)$ is due to the fact that it does not depend on $t$; see below). Using successively the changes of variables $\tilde{u}=u / h, \tilde{v}=v / h$ in the integrals with respect to $u$ and $v$ and $\tilde{\xi}=h \xi$ in the integral with respect to $\xi$, the summand $E_{2}(h)$ can be written 
as

$$
\begin{aligned}
& E_{2}(h)=\alpha_{H} \int_{\mathbb{R}^{d}} \int_{t}^{t+h} \int_{t}^{t+h} \mathcal{F} G_{1}(t+h-u, \cdot)(\xi) \\
& \times \overline{\mathcal{F} G_{1}(t+h-v, \cdot)(\xi)}|u-v|^{2 H-2} d u d v \mu(d \xi) \\
& =\alpha_{H} \int_{\mathbb{R}^{d}} \frac{\mu(d \xi)}{|\xi|^{2}} \int_{0}^{h} \int_{0}^{h} \sin (u|\xi|) \sin (v|\xi|)|u-v|^{2 H-2} d u d v \\
& =\alpha_{H} h^{2 H} \int_{\mathbb{R}^{d}} \frac{\mu(d \xi)}{|\xi|^{2}} \int_{0}^{1} \int_{0}^{1} \sin (u|\xi| h) \sin (v|\xi| h)|u-v|^{2 H-2} d u d v \\
& =\alpha_{H} h^{2 H+2-\beta} \int_{\mathbb{R}^{d}} \frac{\mu(d \xi)}{|\xi|^{2}} \int_{0}^{1} \int_{0}^{1} \sin (u|\xi|) \sin (v|\xi|)|u-v|^{2 H-2} d u d v .
\end{aligned}
$$

We use the following notation:

$$
N_{t}(\xi)=\frac{\alpha_{H}}{|\xi|^{2}} \int_{0}^{t} \int_{0}^{t} \sin (u|\xi|) \sin (v|\xi|)|u-v|^{2 H-2} d u d v, \quad t \in[0, T], \xi \in \mathbb{R}^{d}
$$

By Proposition 3.7 in [2] the term

$$
N_{1}(\xi)=\frac{\alpha_{H}}{|\xi|^{2}} \int_{0}^{1} \int_{0}^{1} \sin (u|\xi|) \sin (v|\xi|)|u-v|^{2 H-2} d u d v
$$

satisfies the inequality

$$
N_{1}(\xi) \leq C_{H}\left(\frac{1}{1+|\xi|^{2}}\right)^{H+1 / 2}
$$

with $C_{H}$ a positive constant not depending on $h$. Consequently the term $E_{2}(h)$ is bounded by

$$
E_{2}(h) \leq C h^{2 H+2-\beta} \int_{\mathbb{R}^{d}}\left(\frac{1}{1+|\xi|^{2}}\right)^{H+1 / 2} \mu(d \xi)
$$

and this is clearly finite due to (2.16). Relations (3.6) and (3.8) give the first part of the conclusion.

Now we analyse now the lower bound of the increments of $u(t, x)$ with respect to the variable $t$. Let $h>0, x \in[-M, M]^{d}$, and $t \in\left[t_{0}, T\right]$ be such that $t+h \in\left[t_{0}, T\right]$. From the decomposition

$$
\begin{aligned}
\mathbf{E}|u(t+h, x)-u(t, x)|^{2}= & \left\|\left(g_{t+h, x}-g_{t, x}\right) 1_{[0, t]}\right\|_{\mathcal{H \mathcal { P }}}^{2}+\left\|g_{t+h, x} 1_{[t, t+h]}\right\|_{\mathcal{H \mathcal { P }}}^{2} \\
& +2\left\langle\left(g_{t+h, x}-g_{t, x}\right) 1_{[0, t]}, g_{t+h, x} 1_{[t, t+h]}\right\rangle_{\mathcal{H P}}
\end{aligned}
$$

we immediately obtain, since the second summand on the right-hand side is positive,

$$
\begin{aligned}
\mathbf{E} \mid u & (t+h, x)-\left.u(t, x)\right|^{2} \\
& \geq\left\|\left(g_{t+h, x}-g_{t, x}\right) 1_{[0, t]}\right\|_{\mathcal{H P}}^{2}+2\left\langle\left(g_{t+h, x}-g_{t, x}\right) 1_{[0, t]}, g_{t+h, x} 1_{[t, t+h]}\right\rangle_{\mathcal{H P}} \\
& :=E_{1, t}(h)+E_{3, t}(h) .
\end{aligned}
$$


We can assume, without loss of the generality, that $t=1 / 2$. Denote $E_{1,1 / 2}(h):=$ $E_{1}(h)$. We first prove that

$$
E_{1}(h) \geq c h^{2 H+1-\beta}-c^{\prime} h^{2 H+2-\beta} .
$$

for $h$ small enough. Recall that we have an exact expression for $E_{1}(h)$ (see (3.4)). Actually,

$$
\begin{aligned}
E_{1}(h)= & \int_{\mathbb{R}^{d}} \frac{d \xi}{|\xi|^{d-\beta+2 H+2}} \sin (h|\xi|)^{2}\left\|\cos (\cdot) 1_{(h|\xi|, h|\xi|+|\xi|)}\right\|_{\mathcal{H}}^{2} \\
= & \alpha_{H} \int_{\mathbb{R}^{d}} \frac{d \xi}{|\xi|^{d-\beta+2 H+2}} \sin (h|\xi|)^{2} \\
& \times \int_{h|\xi|}^{(1+h)|\xi|} \int_{h|\xi|}^{(1+h)|\xi|} d u d v|u-v|^{2 H-2} \cos u \cos v \\
= & \alpha_{H} \int_{\mathbb{R}^{d}} \frac{d \xi}{|\xi|^{d-\beta+2 H+2}} \sin (h|\xi|)^{2} \\
& \times \int_{0}^{|\xi|} \int_{0}^{|\xi|} d u d v \cos (u+h|\xi|) \cos (v+h|\xi|)|u-v|^{2 H-2} .
\end{aligned}
$$

By using the trigonometric formula $\cos (x+y)=\cos (x) \cos (y)-\sin (x) \sin (y)$ we write

$$
\begin{aligned}
E_{1}(h)= & \alpha_{H} \int_{\mathbb{R}^{d}} \frac{d \xi}{|\xi|^{d-\beta+2 H+2}} \sin (h|\xi|)^{2}\left[\cos (h|\xi|)^{2} \int_{0}^{|\xi|} \int_{0}^{|\xi|} d u d v \cos u \cos v|u-v|^{2 H-2}\right. \\
& -2 \sin (h|\xi|) \cos (h|\xi|) \int_{0}^{|\xi|} \int_{0}^{|\xi|} d u d v \sin u \cos v|u-v|^{2 H-2} \\
& \left.+\sin (h|\xi|)^{2} \int_{0}^{|\xi|} \int_{0}^{|\xi|} d u d v \sin u \sin v|u-v|^{2 H-2}\right] \\
:= & A+B+C .
\end{aligned}
$$

We neglect the first term since it is positive. We bound the second term by $c h^{2 H+2-\beta}$. Using again trigonometric identities, Lemma 3.3 (used at the third line below), and the change of variables $\tilde{v}=u-v$ we have

$$
\begin{aligned}
& -2 \sin (h|\xi|) \cos (h|\xi|) \int_{0}^{|\xi|} \int_{0}^{|\xi|} d u d v \sin u \cos v|u-v|^{2 H-2} \\
& =-\sin (h|\xi|) \cos (h|\xi|) \int_{0}^{|\xi|} \int_{0}^{|\xi|} d u d v(\sin (u+v)+\sin (u-v))|u-v|^{2 H-2} \\
& =-\sin (h|\xi|) \cos (h|\xi|) \int_{0}^{|\xi|} \int_{0}^{|\xi|} d u d v \sin (u+v)|u-v|^{2 H-2} \\
& =c \cdot \sin (h|\xi|) \cos (h|\xi|) \int_{0}^{|\xi|} v^{2 H-2}(\cos (2|\xi|-v)-\cos (v)) d v
\end{aligned}
$$


and thus

$$
\begin{aligned}
B=c \cdot \alpha_{H} \int_{\mathbb{R}^{d}} \frac{d \xi}{|\xi|^{d-\beta+2 H+2}} \sin (h|\xi|)^{2} \sin (h|\xi|) \cos (h|\xi|) \\
\quad \times \int_{0}^{|\xi|} v^{2 H-2}(\cos (2|\xi|-v)-\cos (v)) d v \\
=-c \cdot \alpha_{H} \int_{\mathbb{R}^{d}} \frac{d \xi}{|\xi|^{d-\beta+2 H+2}} \sin (h|\xi|)^{3} \cos (h|\xi|) \sin (|\xi|) \int_{0}^{|\xi|} v^{2 H-2} \sin (|\xi|-v) d v \\
=-c \cdot \alpha_{H} \int_{\mathbb{R}^{d}} \frac{d \xi}{|\xi|^{d-\beta+2 H+2}} \sin (h|\xi|)^{3} \cos (h|\xi|) \sin (|\xi|) \\
\quad \times \int_{0}^{|\xi|} v^{2 H-2}(\sin (|\xi|) \cos (v)-\cos (|\xi|) \sin (v)) d v \\
=-c \cdot \alpha_{H} \int_{\mathbb{R}^{d}} \frac{d \xi}{|\xi|^{d-\beta+2 H+2}} \sin (h|\xi|)^{3} \cos (h|\xi|) \sin (|\xi|) \\
\quad \times\left(\sin (|\xi|) \int_{0}^{|\xi|} v^{2 H-2} \cos (v) d v-\cos (|\xi|) \int_{0}^{|\xi|} v^{2 H-2} \sin (v) d v\right) \\
=-c \cdot \alpha_{H} \int_{|\xi| \leq 1} \frac{d \xi}{|\xi|^{d-\beta+2 H+2}} \sin (h|\xi|)^{3} \cos (h|\xi|) \sin (|\xi|) \\
\quad \times\left(\sin (|\xi|) \int_{0}^{|\xi|} v^{2 H-2} \cos (v) d v-\cos (|\xi|) \int_{0}^{|\xi|} v^{2 H-2} \sin (v) d v\right) \\
-c \cdot \alpha_{H} \int_{|\xi| \geq 1} \frac{d \xi}{|\xi|^{d-\beta+2 H+2}} \sin (h|\xi|)^{3} \cos (h|\xi|) \sin (|\xi|) \\
\left.\quad \times \int_{0}^{|\xi|} v^{2 H-2} \cos (v) d v-\cos (|\xi|) \int_{0}^{|\xi|} v^{2 H-2} \sin (v) d v\right) .
\end{aligned}
$$

Taking absolute value we see that the part over the set $|\xi| \leq 1$ is bounded by $c h^{3}$ by simply majorizing $\sin (h|\xi|)$ by $h|\xi|, \cos (h|\xi|) \sin (|\xi|)$ by 1 , and

$$
\left|\sin (|\xi|) \int_{0}^{|\xi|} v^{2 H-2} \cos (v) d v-\cos (|\xi|) \int_{0}^{|\xi|} v^{2 H-2} \sin (v) d v\right|
$$

by a constant. Concerning the part over the region $|\xi| \geq 1$ we bound again the last expression by a constant and we use the change of variables $\tilde{\xi}=h \xi$. This part is bounded by

$$
\begin{aligned}
& h^{2 H+2-\beta} \int_{|\xi| \geq h} \frac{d \xi}{|\xi|^{d-\beta+2 H+2}} \mid \sin (|\xi|)^{3} \cos (|\xi|) \sin (|\xi| / h) \mid \\
& \leq h^{2 H+2-\beta} \int_{\mathbb{R}^{d}} \frac{d \xi}{|\xi|^{d-\beta+2 H+2}}\left|\sin (|\xi|)^{3}\right| \leq c h^{2 H+2-\beta}
\end{aligned}
$$

since the last integral is convergent at infinity by bounding the sine function by 1 and at zero by bounding $\sin (x)$ by $x$ and using the assumption $\beta>2 H-1$. Therefore

$$
B \leq c h^{2 H+2-\beta} .
$$


Now we bound summand $C$ from below. In this summand the $\mathcal{H}$ norm of the sine function appears and this has been analyzed in [2]. We have, after the change of variables $\tilde{u}=u /|\xi|, \tilde{v}=v /|\xi|$,

$$
\begin{aligned}
C & =\alpha_{H} \int_{\mathbb{R}^{d}} \frac{d \xi}{|\xi|^{d-\beta+2}} \sin (h|\xi|)^{4} \int_{0}^{1} \int_{0}^{1} \sin (u|\xi|) \sin (v|\xi|)|u-v|^{2 H-2} d u d v \\
& \geq \alpha_{H} \int_{|\xi| \geq 1} \frac{d \xi}{|\xi|^{d-\beta+2}} \sin (h|\xi|)^{4} \int_{0}^{1} \int_{0}^{1} \sin (u|\xi|) \sin (v|\xi|)|u-v|^{2 H-2} d u d v .
\end{aligned}
$$

We will use Proposition 3.8 in [2] (more precisely, we will use the inequality (34) in that paper with $k=0$; we notice that the term $\sin (h|\xi|)^{4}$ does not appear in this proof, but analyzing the proof step-by-step we can see that this term can be added without problems). For $h$ small, we have that,

$$
\begin{aligned}
C \geq \alpha_{H} & \int_{|\xi| \geq 1} \frac{d \xi}{|\xi|^{d-\beta}} \sin (h|\xi|)^{4} \frac{1}{|\xi|^{2}} \int_{0}^{1} \int_{0}^{1} \sin (u|\xi|) \sin (v|\xi|)|u-v|^{2 H-2} d u d v \\
& \geq \alpha_{H} \int_{|\xi| \geq 1} \frac{d \xi}{|\xi|^{d-\beta}} \sin (h|\xi|)^{4} \frac{1}{|\xi|^{2 H+1}} \\
& =\alpha_{H} h^{2 H+1-\beta} \int_{|\xi| \geq h} \frac{d \xi}{|\xi|^{d-\beta+2 H+1}} \sin (|\xi|)^{4} \\
& \geq \alpha_{H} h^{2 H+1-\beta} \int_{|\xi| \geq 1} \frac{d \xi}{|\xi|^{d-\beta+2 H+1}} \sin (|\xi|)^{4} \\
(3.11) \quad & =c \cdot \alpha_{H} h^{2 H+1-\beta} .
\end{aligned}
$$

Relations (3.10) and (3.11) imply (3.9). Now, from the relation (3.9), for every $t_{0} \leq s<t<T$ with $s$ and $t$ close enough

$$
E_{1}(t-s) \geq c(t-s)^{2 H+1-\beta}-c^{\prime}(t-s)^{2 H+2-\beta} \geq \frac{c}{2}(t-s)^{2 H+1-\beta}
$$

if $|t-s| \leq c /\left(2 c^{\prime}\right)$. To extend the above inequality to arbitrary values of $|t-s|$, we proceed as in the proof of Proposition 4.1 in [11]. Notice that the function $g(t, s, x, y):=\mathbf{E}|u(t, x)-u(s, x)|^{2}$ is positive and continuous with respect to all its arguments and therefore it is bounded below on the set $\left\{(t, s, x, y) \in\left[t_{0}, T\right]^{2} \times\right.$ $\left.[-M, M]^{2 d} ;|t-s| \geq \varepsilon\right\}$ by a constant depending on $\varepsilon>0$. Hence for $|t-s| \geq \frac{c}{2 c^{\prime}}$ there also holds

$$
E_{1}(t-s) \geq c_{1}|t-s|^{2 H+1-\beta} .
$$

On the other hand, from (3.3), (3.8), and Cauchy-Schwarz inequality, we obtain

$$
\begin{aligned}
& E_{3, t}(h)=\left\langle\left(g_{t+h, x}-g_{t, x}\right) 1_{[0, t]}, g_{t+h, x} 1_{[t, t+h]}\right\rangle_{\mathcal{H P}} \\
& \quad \leq\left\|\left(g_{t+h, x}-g_{t, x}\right) 1_{[0, t]}\right\|_{\mathcal{H P}}\left\|g_{t+h, x} 1_{[t, t+h]}\right\|_{\mathcal{H P}} \leq c h^{(2 H+1-\beta) / 2+(2 H+2-\beta) / 2} .
\end{aligned}
$$

Consequently,

$$
\mathbf{E}|u(t+h, x)-u(t, x)|^{2} \geq C h^{2 H+1-\beta}-C^{\prime} h^{(2 H+1-\beta) / 2+(2 H+2-\beta) / 2},
$$


and this implies that, for every $s, t \in\left[t_{0}, T\right]$ and $x \in[-M, M]^{d}$,

$$
\mathbf{E}|u(t, x)-u(s, x)|^{2} \geq \frac{C}{2}|t-s|^{2 H+1-\beta} \quad \text { if }|t-s| \leq\left(\frac{C}{2 C^{\prime}}\right)^{1 / 2} .
$$

Similarly, as above, the previous inequality can be extended to arbitrary values of $s, t \in\left[t_{0}, T\right]$.

Proposition 3.4 implies the following Hölder property for the solution to (2.13).

Corollary 3.5. Assume (3.1). Then for every $x \in \mathbb{R}^{d}$ the map

$$
t \rightarrow u(t, x)
$$

is almost surely Hölder continuous of order $\delta \in(0,(2 H+1-\beta) / 2)$.

Proof. This is a consequence of the relations (3.3) and (3.8) in the proof of Proposition 3.4 and the fact that $u$ is Gaussian.

Remark 3.6. (i) Following the proof of Theorem 5.1 in $[10]$ we can show that the mapping $t \rightarrow u(t, x)$ is not Hölder continuous of order $(2 H+1-\beta) / 2$.

(ii) When $H=1 / 2$, we recover the results in [10] and [11] in the linear case.

\subsection{Regularity in space}

Let us discuss the behavior of the solution $u$ to the equation (2.13) with respect to the spatial variable. We have:

Proposition 3.7. Assume (3.1) and fix $M>0$ and $t \in\left[t_{0}, T\right]$. Then there exist positive constants $c_{3}$ and $c_{4}$ such that for any $x, y \in[-M, M]^{d}$

$$
c_{3}|x-y|^{2 H+1-\beta} \leq \mathbf{E}|u(t, x)-u(t, y)|^{2} \leq c_{4}|x-y|^{2 H+1-\beta} .
$$

Proof. Let $z \in \mathbb{R}^{d}$. We compute

$$
\begin{aligned}
& \mathbf{E}|u(t, x+z)-u(t, x)|^{2}=\left\|g_{t, x+z}-g_{t, x}\right\|_{\mathcal{H P}}^{2} \\
& \begin{aligned}
=\alpha_{H} \int_{\mathbb{R}^{d}} \int_{0}^{t} \int_{0}^{t} \mathcal{F}\left(g_{t, x+z}-g_{t, x}\right)(u, \cdot)(\xi) \overline{\mathcal{F}\left(g_{t, x+z}-g_{t, x}\right)(v, \cdot)(\xi)} \\
\quad \times|u-v|^{2 H-2} d u d v \mu(d \xi)
\end{aligned} \\
& =\alpha_{H} \int_{0}^{t} \int_{0}^{t}|u-v|^{2 H-2} d u d v \int_{\mathbb{R}^{d}}\left|e^{-i \xi \cdot(x+z)}-e^{-i \xi \cdot x}\right|^{2} \mathcal{F} G_{1}(u, \cdot)(\xi) \overline{\mathcal{F} G_{1}(v, \cdot)(\xi)} \mu(d \xi) \\
& =\alpha_{H} \int_{0}^{t} \int_{0}^{t}|u-v|^{2 H-2} d u d v \int_{\mathbb{R}^{d}}\left|e^{-i \xi \cdot z}-1\right|^{2} \frac{\sin (u|\xi|)}{|\xi|} \cdot \frac{\sin (v|\xi|)}{|\xi|} \mu(d \xi) \\
& =: E_{1, x}(z)+E_{2, x}(z),
\end{aligned}
$$

where $E_{1, x}(z)$ and $E_{2, x}(z)$ are the integrals over the regions $|\xi|<1$ and $|\xi| \geq 1$, respectively. 
For the first expression is easy to see that, using the inequality $\left|1-e^{-i \xi z}\right|^{2} \leq$ $|\xi|^{2}|z|^{2}$, we get the bound

$$
E_{1, x}(z) \leq C|z|^{2} \int_{|\xi| \leq 1} \mu(d \xi)
$$

Developing the second expression we get

$$
\begin{aligned}
& E_{2, x}(z)=\alpha_{H} \int_{0}^{t} \int_{0}^{t}|u-v|^{2 H-2} d u d v \int_{|\xi| \geq 1}\left|e^{-i \xi \cdot z}-1\right|^{2} \frac{\sin (u|\xi|)}{|\xi|} \cdot \frac{\sin (v|\xi|)}{|\xi|} \mu(d \xi) \\
& =2 \alpha_{H} \int_{0}^{t} \int_{0}^{t}|u-v|^{2 H-2} d u d v \int_{|\xi| \geq 1} \frac{d \xi}{|\xi|^{d-\beta}}(1-\cos (z \cdot \xi)) \frac{\sin (u|\xi|)}{|\xi|} \cdot \frac{\sin (v|\xi|)}{|\xi|}
\end{aligned}
$$

where $z \cdot \xi$ means the scalar product in $\mathbb{R}^{d}$. Again from Proposition 3.7 in [2] we have that

$$
N_{t}(\xi) \leq c_{t, H}\left(\frac{1}{1+|\xi|^{2}}\right)^{H+1 / 2}
$$

for any $t>0,|\xi| \geq 1$, where $N_{t}(\xi)$ is given by (3.7). Hence, writing, $e=z /|z|$

$$
\begin{aligned}
E_{2, x}(z) & \leq C \int_{\mathbb{R}^{d}} \frac{d \xi}{|\xi|^{d-\beta}}(1-\cos (z \cdot \xi))\left(\frac{1}{1+|\xi|^{2}}\right)^{H+1 / 2} \\
& =C z^{2 H+1-\beta} \int_{\mathbb{R}^{d}} \frac{d w}{|w|^{d-\beta}}(1-\cos (w \cdot e))\left(\frac{1}{|w|^{2}+|z|^{2}}\right)^{H+1 / 2} \\
& \leq C|z|^{2 H+1-\beta}
\end{aligned}
$$

where we used the change of variables $w=\xi|z|$. This proves the upper bound.

Let us prove the sharpness of this bound (i.e., the lower bound). We can assume, without loss of generality, that $t=1$. We note that

$$
\begin{aligned}
& \mathbf{E}|u(1, x+z)-u(1, x)|^{2} \geq F_{2}(z) \\
& :=2 \alpha_{H} \int_{0}^{1} \int_{0}^{1} d u d v|u-v|^{2 H-2} \int_{|\xi| \geq 1} \frac{d \xi}{|\xi|^{d-\beta}}(1-\cos (\xi \cdot z)) \frac{\sin (u|\xi|)}{|\xi|} \cdot \frac{\sin (v|\xi|)}{|\xi|} .
\end{aligned}
$$

Condition (2.17) implies that

$$
\int_{|\xi| \geq 1} \frac{\mu(d \xi)}{|\xi|^{3}} \leq \int_{|\xi| \geq 1} \frac{\mu(d \xi)}{|\xi|^{2 H+1}}<\infty
$$

We apply Proposition 3.8 in [2] (more precisely, the inequality (34) in [2] with $k=0$ ) and we get (note that the result in [2] is stated without the factor $(1-\cos (\xi \cdot z))$ but by analyzing the proof we can see that this factor may be added without problems):

$$
F_{2}(z) \geq C \int_{|\xi| \geq 1} \frac{d \xi}{|\xi|^{d-\beta+2 H+1}}(1-\cos (\xi \cdot z))-C^{\prime} \int_{|\xi| \geq 1} \frac{d \xi}{|\xi|^{d-\beta+2 H+2}}(1-\cos (\xi \cdot z))
$$


(here $C>C^{\prime}$ ) and, by the change of variables $\xi|z|=w$ in the integral with respect to $\xi$,

$$
\begin{aligned}
F_{2}(z) \geq & C z^{2 H+1-\beta} \int_{|w| \geq|z|} \frac{d w}{|w|^{d-\beta+2 H+1}}(1-\cos (w \cdot e)) \\
& -C^{\prime} z^{2 H+2-\beta} \int_{|w| \geq|z|} \frac{d w}{|w|^{d-\beta+2 H+2}}(1-\cos (w \cdot e)) \\
\geq & C z^{2 H+1-\beta} .
\end{aligned}
$$

As in the proof of Theorem 5.1 in [10], we obtain that the integral

$$
\int_{|w| \geq|z|} \frac{d w}{|w|^{d-\beta+2 H+1}}(1-\cos (w \cdot e))
$$

is bounded below by a constant. (Notice that $\beta>2 H-1$ implies that the first integral above is convergent when $z$ is zero, because $1-\cos (x) \approx x^{2}$, when $x$ is near 0 ). Thus, it is immediate that

$$
\mathbf{E}|u(1, x+z)-u(1, x)|^{2} \geq C z^{2 H+1-\beta} .
$$

We have the following result concerning the Hölder continuity in space. We mention that it is little more than an extension of Proposition 3.7.

Proposition 3.8. Assume $\beta \in(0, d \wedge(2 H+1))$. Then for any $t \in\left[t_{0}, T\right]$ the map

$$
x \rightarrow u(t, x)
$$

is almost surely Hölder continuous of order $\delta \in\left(0,\left(\frac{2 H+1-\beta}{2}\right) \wedge 1\right)$.

Proof. We claim that

$$
\mathbf{E}|u(t, x)-u(t, y)|^{2} \leq c|x-y|^{(2 H+1-\beta) \wedge 2}
$$

whenever $|x-y|$ is sufficiently small. From Proposition 3.7, (3.13) is true when $\beta>2 H-1$. When $\beta \in(0,2 H-1]$ then it suffices to consider the part of the quantity $\mathbf{E}|u(t, x+z)-u(t, x)|^{2}$ over the region $|\xi| \leq 1$ (the part over the region $|\xi|>1$ is, as in the proof of Proposition 3.7, bounded by $c z^{2 H+1-\beta}$ so by $c z^{2}$ for $z$ small). It is immediate to see that, using the inequality $\left|1-e^{-i \xi z}\right|^{2} \leq|\xi|^{2}|z|^{2}$ the considered part is less than $C|z|^{2} \int_{|\xi| \leq 1} \mu(d \xi)$. This concludes the proof of (3.13).

The conclusion is a consequence of Proposition 3.7, the Gaussian nature of $u$ and the Kolmogorov continuity theorem.

Remark 3.9. (i) When $H=1 / 2$, the above result coincides with the results of [10] and [11]).

(ii) In Proposition 3.8 we distinguish two cases: if $\beta \in(0,2 H-1)$ then the solution to (2.13) has spatial Hölder continuity of order $\alpha$ for every $\alpha \in(0,1)$ while if $\beta \in(2 H-1, d \wedge(2 H+1))$ the Hölder exponent is $\delta \in(0,(2 H+1-\beta) / 2)<1$. 
(iii) There is another way to see why the cases $\beta \in(0,2 H-1]$ and $\beta \in$ $(2 H-1, d \wedge(2 H+1))$ need to be separated. Define

$$
\begin{aligned}
g_{t}(z):= & \mathbf{E}|u(t, x+z)-u(t, x)|^{2} \\
= & 2 \alpha_{H} \int_{0}^{t} \int_{0}^{t}|u-v|^{2 H-2} d u d v \\
& \times \int_{\mathbb{R}^{d}} \frac{d \xi}{|\xi|^{d-\beta}}(1-\cos (z \cdot \xi)) \frac{\sin (u|\xi|)}{|\xi|} \cdot \frac{\sin (v|\xi|)}{|\xi|} .
\end{aligned}
$$

We study the behavior of $g_{t}$ around $z=0$. We also assume that $d=1$. Notice first that $g_{t}(0)=0$ and

$$
g_{t}^{\prime}(z)=2 \alpha_{H} \int_{0}^{t} \int_{0}^{t}|u-v|^{2 H-2} d u d v \int_{\mathbb{R}^{d}} \frac{d \xi}{|\xi|^{d-\beta-1}} \sin (z \cdot \xi) \frac{\sin (u|\xi|)}{|\xi|} \cdot \frac{\sin (v|\xi|)}{|\xi|}
$$

and thus $g_{t}^{\prime}(0)=0$ provided that $\beta<2 H$. Moreover

$$
g_{t}^{\prime \prime}(z)=2 \alpha_{H} \int_{0}^{t} \int_{0}^{t}|u-v|^{2 H-2} d u d v \int_{\mathbb{R}^{d}} \frac{d \xi}{|\xi|^{d-\beta-2}} \cos (z \cdot \xi) \frac{\sin (u|\xi|)}{|\xi|} \cdot \frac{\sin (v|\xi|)}{|\xi|}
$$

and

$$
\begin{aligned}
g_{t}^{\prime \prime}(0) & =2 \alpha_{H} \int_{0}^{t} \int_{0}^{t}|u-v|^{2 H-2} d u d v \int_{\mathbb{R}^{d}} \frac{d \xi}{|\xi|^{d-\beta-2}} \frac{\sin (u|\xi|)}{|\xi|} \cdot \frac{\sin (v|\xi|)}{|\xi|} \\
& \leq C_{t} 2 \alpha_{H} \int_{\mathbb{R}^{d}} \frac{d \xi}{|\xi|^{d-\beta-2}}\left(\frac{1}{1+|\xi|^{2}}\right)^{H+1 / 2},
\end{aligned}
$$

which is a finite constant for $\beta<2 H-1$. Therefore $g_{t}(z)$ behaves as $C z^{2}$ for $z$ close to 0 .

\subsection{Joint regularity}

We denote by $\Delta$ the metric

$$
\Delta((t, x) ;(s, y))=|t-s|^{2 H+1-\beta}+|x-y|^{2 H+1-\beta},
$$

on $[0, T] \times \mathbb{R}^{d}$. From Propositions 3.4 and 3.7, we obtain the following result.

Theorem 3.10. Fix $M>0$ and assume (3.1). For every $t, s \in\left[t_{0}, T\right]$ and $x, y \in$ $[-M, M]^{d}$ there exist positive constants $C_{1}$ and $C_{2}$ such that

$$
C_{1} \Delta((t, x) ;(s, y)) \leq \mathbf{E}|u(t, x)-u(s, y)|^{2} \leq C_{2} \Delta((t, x) ;(s, y)) .
$$

Proof. The upper bound can be easily obtained by using the upper bounds in Propositions 3.4 and 3.7 since

$$
\begin{aligned}
\mathbf{E}|u(t, x)-u(s, y)|^{2} & \leq 2 \mathbf{E}|u(t, x)-u(s, x)|^{2}+2 \mathbf{E}|u(s, x)-u(s, y)|^{2} \\
& \leq C_{2}\left(|t-s|^{2 H+1-\beta}+|x-y|^{2 H+1-\beta}\right) .
\end{aligned}
$$


Concerning the lower bound, it suffices to follow the lines of the proof of Lemma 2.1 in [18] (see also steps 3 and 4 in the proof of Proposition 4.1 in [11]). We will briefly explain the main points of the proof. The proof needs to be divided into three cases:

$$
\begin{aligned}
& |t-s|^{2 H+1-\beta} \leq \frac{c_{3}}{4 c_{2}}|x-y|^{2 H+1-\beta}, \\
& |t-s|^{2 H+1-\beta} \geq \frac{4 c_{4}}{c_{1}}|x-y|^{2 H+1-\beta}, \\
& \text { and } \quad \frac{4 c_{4}}{c_{1}}|x-y|^{2 H+1-\beta} \geq|t-s|^{2 H+1-\beta} \geq \frac{c_{3}}{4 c_{2}}|x-y|^{2 H+1-\beta},
\end{aligned}
$$

with the constants $c_{1}, c_{2}, c_{3}$, and $c_{4}$ appearing in the statements of Propositions 3.4 and 3.7. The first case can be handled as follows:

$$
\begin{aligned}
\mathbf{E} \mid u(t, x) & -\left.u(s, y)\right|^{2} \geq \frac{1}{2} \mathbf{E}|u(t, x)-u(t, y)|^{2}-\mathbf{E}|u(t, y)-u(s, y)|^{2} \\
& \geq \frac{1}{2} c_{3}|x-y|^{2 H+1-\beta}-c_{2}|t-s|^{2 H+1-\beta} \\
& \geq \frac{1}{2} c_{3}|x-y|^{2 H+1-\beta}-\frac{1}{4} c_{3}|x-y|^{2 H+1-\beta} \\
& =\frac{1}{4} c_{3}|x-y|^{2 H+1-\beta} \geq \frac{c_{3}}{8}|x-y|^{2 H+1-\beta}+\frac{c_{3}}{8} \frac{4 c_{2}}{c_{3}}|t-s|^{2 H+1-\beta} \\
& \geq C_{1} \Delta((t, x) ;(s, y)) .
\end{aligned}
$$

The other cases follow in a similar way from Lemma 3.1 in [18], replacing the exponents in [18] with the exponents used here.

Remark 3.11. The result of Theorem 3.10 can be stated also in the following form. Fix $M>0$ and assume (3.1). For every $t, s \in\left[t_{0}, T\right]$ and $x, y \in[-M, M]^{d}$ with $(t, x)$ close enough to $(s, y)$, there exist positive constants $C_{1}$, and $C_{2}$ such that $C_{1}(|t-s|+|x-y|)^{2 H+1-\beta} \leq \mathbf{E}|u(t, x)-u(s, y)|^{2} \leq C_{2}(|t-s|+|x-y|)^{2 H+1-\beta}$.

\section{Hitting times}

We discuss the upper and lower bounds for the hitting probabilities of the solution $u$ to equation (2.13). These bounds will be given in terms of the Newtonian capacity and the Hausdorff measure of the set (see Section 2 for the definition). We recall our notation: if $V=\left(V(x), x \in \mathbb{R}^{m}\right)$ is an $\mathbb{R}^{k}$-valued stochastic process then $V(S)$ denotes the range of the Borel set $S$ under the random mapping $x \rightarrow V(x)$.

Our result is based on the following criteria for the hitting probabilities proved as Theorem 2.1 of [4].

Theorem 4.1. Let $X=X(t), t \in \mathbb{R}^{N}$, be an $\mathbb{R}^{k}$-valued centered Gaussian process and fix $I \subset \mathbb{R}^{N}$. Assume that there exist positive constants $a_{1}, a_{2}, a_{3}$ and $a_{4}$ such that

(i) For every $t \in I, \mathbf{E}\left[X(t)^{2}\right] \geq a_{1}>0$. 
(ii) There exists $\alpha_{1}, \ldots, \alpha_{N} \in(0,1)$ such that for all $t=\left(t_{1}, \ldots, t_{N}\right), s=$ $\left(s_{1}, \ldots, s_{N}\right) \in I$ there holds

$$
a_{2} \sum_{j=1}^{N}\left|t_{j}-s_{j}\right|^{2 \alpha_{j}} \leq \mathbf{E}|X(t)-X(s)|^{2} \leq a_{3} \sum_{j=1}^{N}\left|t_{j}-s_{j}\right|^{2 \alpha_{j}} .
$$

(iii) For all $t=\left(t_{1}, \ldots, t_{N}\right), s=\left(s_{1}, \ldots, s_{N}\right) \in I$,

$$
\operatorname{Var}(X(t) \mid X(s)) \geq a_{4} \sum_{j=1}^{N}\left|t_{j}-s_{j}\right|^{2 \alpha_{j}}
$$

Then there exist positive constants $a_{5}$ and $a_{6}$ such that for every Borel set $A$ in $\mathbb{R}^{k}$

$$
a_{5} \operatorname{Cap}_{k-Q}(A) \leq P(X(I) \cap A \neq \emptyset) \leq a_{6} \mathcal{H}_{k-Q}(A)
$$

where $Q=\sum_{j=1}^{N} 1 / \alpha_{j}$.

Next, we will show that the solution to (2.13) satisfies the assumptions of the previous result. This will be accomplished via several lemmas.

Lemma 4.2. Assume (3.1) and let $u$ be the solution to (2.13). Then for every $t \in\left[t_{0}, T\right]$ and $x \in \mathbb{R}^{d}$

$$
\mathbf{E} u(t, x)^{2} \geq C
$$

Proof. Let $\sigma_{t, x}^{2}$ be the variance of $u(t, x)$. We need to bound this variance from below. Assume for simplicity that $t=1$. Then

$$
\begin{aligned}
\sigma_{1, x}^{2} & =\mathbf{E}|u(1, x)|^{2}=\alpha_{H} \int_{0}^{1} \int_{0}^{1} d u d v|u-v|^{2 H-2} \int_{\mathbb{R}^{d}} \frac{d \xi}{|\xi|^{d-\beta+2}} \sin (u|\xi|) \sin (v|\xi|) \\
& \geq \alpha_{H} \int_{0}^{1} \int_{0}^{1} d u d v|u-v|^{2 H-2} \int_{|\xi| \leq 1} \frac{d \xi}{|\xi|^{d-\beta+2}} \sin (u|\xi|) \sin (v|\xi|) \\
& \geq \alpha_{H} \sin ^{2} 1 \int_{0}^{1} \int_{0}^{1} d u d v|u-v|^{2 H-2} u v=C>0
\end{aligned}
$$

where we used that $\sin x \geq x \sin 1$ for every $x \in[0,1]$. The general case $t \in\left[t_{0}, T\right]$ follows in the same way by making the change of variables $\tilde{u}=u / t, \tilde{v}=v / t$ and then working on the domain $D=\left\{\xi \in \mathbb{R}^{d},|\xi| \leq 1 /(u t)\right\}$.

Now, we bound the conditional variance (condition iii in Theorem 4.1).

Lemma 4.3. Assume (3.1) and fix $t_{0}, M>0$. Then for every $s, t \in\left[t_{0}, T\right]$ and $x, y \in[-M, M]^{d}$,

$$
\operatorname{Var}(u(t, x) \mid u(s, y)) \geq C \Delta((t, x) ;(s, y))
$$

where $\Delta$ is the metric given by (3.14). 
Proof. We will use the following formula: if $(U, V)$ is a centered Gaussian vector, then

$$
\operatorname{Var}(U, V)=\frac{\left(\rho_{U, V}^{2}-\left(\sigma_{U}-\sigma_{V}\right)^{2}\right)\left(\left(\sigma_{U}+\sigma_{V}\right)^{2}-\rho_{U, V}^{2}\right)}{4 \sigma_{V}^{2}}
$$

where

$$
\rho_{U, V}^{2}=\mathbf{E}(U-V)^{2}, \quad \sigma_{U}^{2}=\mathbf{E} U^{2}, \quad \text { and } \quad \sigma_{V}^{2}=\mathbf{E} V^{2}
$$

Define

$$
\rho_{t, x, s, y}^{2}=\mathbf{E}|u(t, x)-u(s, y)|^{2}, \quad \sigma^{2}(t, x)=\mathbf{E} u(t, x)^{2}, \quad \sigma_{s, y}^{2}=\mathbf{E} u(s, y)^{2} .
$$

It suffices to show that

$$
\left(\rho_{t, x, s, y}^{2}-\left(\sigma_{t, x}-\sigma_{s, y}\right)^{2}\right)\left(\left(\sigma_{t, x}+\sigma_{s, y}\right)^{2}-\rho_{t, x, s, y}^{2}\right) \geq c \Delta((t, x) ;(s, y))
$$

for every $s, t \in\left[t_{0}, T\right]$ and $x, y \in[-M, M]^{d}$. By Theorem 3.10 the second factor on the left-hand side above is bounded below by a constant. So it remains to check that

$$
\left(\rho_{t, x, s, y}^{2}-\left(\sigma_{t, x}-\sigma_{s, y}\right)^{2}\right) \geq c \Delta((t, x) ;(s, y)),
$$

but this has been done in the proof of Proposition 3.2 in [18] (see also the proof of Lemma 4.3 in [8]).

Remark 4.4. Using the previous result we can bound the joint density $p_{t, x, s, y}$ of the vector $(u(t, x), u(s, y))$. Actually, one can show that for every $t \in\left[t_{0}, T\right]$ and $x, y \in[-M, M]^{d}$ we have the inequality

$$
p_{t, s, x, y}\left(z_{1}, z_{2}\right) \leq C_{1} \Delta((t, x) ;(s, y))^{-k / 2} \exp \left(-\frac{C_{2}\left|z_{1}-z_{2}\right|^{2}}{\Delta(t, x) ;(s, y))}\right)
$$

for every $z_{1}, z_{2} \in[-N, N]^{k}$, where $\Delta$ is the metric defined by (3.14). It suffices to follow the lines of Proposition 3.2 in [18].

We can state now the main result of this section.

Theorem 4.5. Assume (3.1) and consider nontrivial compact sets $I$ and $J$ in $\left[t_{0}, T\right]$ and $[-M, M]^{d}$, respectively. Fix $N>0$ and let $u$ be the solution to the system (1.1). Then for every Borel set A contained in $[-N, N]^{k}$ there holds

$$
C^{-1} \operatorname{Cap}_{k-\gamma}(A) \leq P(u(I \times J) \cap A \neq \emptyset) \leq C \mathcal{H}_{k-\gamma}(A)
$$

with

$$
\gamma=k-\frac{2(d+1)}{2 H+1-\beta}
$$

Proof. The proof is a consequence of Theorem 4.1 and of Lemmas 4.2 and 4.3.

Remark 4.6. (i) Of course, for $H=1 / 2$, our result recovers the results in [11] in the linear case. 
(ii) It is also possible to give some results concerning the probability that, for fixed $t$ and $x$, the sets $u(\{t\} \times J)$ and $u(I \times\{x\})$ hit a given Borel set $A$ contained in $[-N, N]^{k}$, (as before $I$ and $J$ are nontrivial compact sets in $\left[t_{0}, T\right]$ and $[-M, M]^{d}$, respectively). Actually, by routine arguments we have

$$
C^{-1} \operatorname{Cap}_{k-\frac{2 d}{2 H+1-\beta}}(A) \leq P(u(\{t\} \times J) \cap A \neq \emptyset) \leq C \mathcal{H}_{k-\frac{2 d}{2 H+1-\beta}}(A)
$$

and

$$
C^{-1} \operatorname{Cap}_{k-\frac{2}{2 H+1-\beta}}(A) \leq P(u(I \times\{x\}) \cap A \neq \emptyset) \leq C \mathcal{H}_{k-\frac{2}{2 H+1-\beta}}(A) .
$$

Acknowledgements. The second author is grateful to Professors Robert Dalang, Davar Khosnevisan, and Marta Sanz-Solé for useful discussions.

\section{References}

[1] Balan, R. M. And Tudor, C. A.: The stochastic heat equation with fractionalcolored noise: existence of the solution. ALEA Lat. Am. J. Probab. Math. Stat. 4 (2008), 57-87. Erratum in ALEA Lat. Am. J. Probab. Math. Stat. 6 (2009), 343-347.

[2] Balan, R. M. And Tudor, C.A.: The stochastic wave equation with fractional noise: a random field approach. Stochastic Process. Appl. 120 (2010), no. 12, $2468-2494$.

[3] Bayraktar, E., Poor, V. and Sircar, R.: Estimating the fractal dimension of the SP 500 index using wavelet analysis. Int. J. Theor. Appl. Finance 7 (2004), no. 5, 615-643.

[4] Biermé, H., Lacaux, C. And Xiao, Y.: Hitting probabilities and the Hausdorff dimension of the inverse images of anisotropic Gaussian random fields. Bull. Lond. Math. Soc. 41 (2009), no. 2, 253-273.

[5] del Castillo-Negrete, D., Carreras, B. A. And Lynch, V. E.: Front dynamics in reaction-diffusion systems with Levy flights: a fractional difussion approach. Phys. Rev. Letters 91 (2003).

[6] Dalang, R. C.: Extending the martingale measure stochastic integral with applications to spatially homogeneous SPDE's. Electr. J. Probab. 4 (1999), no. 6, 1-29. Erratum in Electr. J. Probab. 6 (2001), no. 6, 1-5.

[7] Dalang, R. C. And Nualart, E.: Potential theory for hyperbolic SPDEs. Ann. Probab. 32 (2004), no. 3A, 2099-2148.

[8] Dalang, R. C., Khosnevisan, D. and Nualart, E. : Hitting probabilities for systems of non-linear heat equations with additive noise. ALEA Lat. Am. J. Probab. Math. Stat. 3 (2007), 231-271.

[9] Dalang, R. C., Khosnevisan, D. and Nualart, E.: Hitting probabilities for systems of non-linear stochastic heat equations with multiplicative noise. Probab. Theory Related Fields 144 (2009), no. 3-4, 371-427.

[10] Dalang, R. And Sanz-Solé, M.: Hölder-Sobolev regularity of the solution to the stochastic wave equation in dimension three. Mem. Amer. Math. Soc. 199 (2009), no. $931,1-70$. 
[11] Dalang, R. And Sanz-Solé, M.: Criteria for hitting probabilities with applications to systems of stochastic wave equations. Bernoulli 16 (2010), no. 4, 1343-1368.

[12] Denk, G., Meintrup, D. And Schaffer, S.: Transient noise simulation: modeling ans simulation of $1 / f$-noise. In Modeling, simulation, and optimization of integrated circuits (Oberwolfach, 2001), 251-267. Internat. Ser. Numer. Math. 146, Birkaäuser, Basel, 2003.

[13] Gubinelli, M., Lejay, A. And Tindel, S.: Young integrals and SPDE's. Potential Anal. 25 (2006), no. 4, 307-326.

[14] Kou, S. C. And Sunney, X.: Generalized Langevin equation with fractional Gaussian noise: subdiffusion within a single protein molecule. Phys. Rev. Letters 93 (2004).

[15] Maslovski, B. And Nualart, D.: Evolution equations driven by a fractional Brownian motion. J. Funct. Anal. 202 (2003), no. 1, 277-305.

[16] Mueller, C. AND Tribe, C.: Hitting probabilities of the random string. Electron. J. Probab. 7 (2002), no. 10, 1-29.

[17] Nualart, D. And Vuillermont, P. A.: Variational solutions for partial differential equations driven by a fractional noise. J. Funct. Anal. 232 (2006), no. 2, 390-454.

[18] Nualart, E. And Viens, F.: The fractional stochastic heat equation on the circle: time regularity and potential theory. Stochastic Process. Appl. 119 (2009), no. 5, $1505-1540$.

[19] Quer-Sardanyons, L. And Tindel, S.: The 1-d stochastic wave equation driven by a fractional Brownian sheet. Stochastic Process. Appl. 117 (2007), no. 10, 1448-1472.

[20] Tindel, S., Tudor, C. A. And Viens, F.: Stochastic evolution equations with fractional Brownian motion. Probab. Theory Related Fields 127 (2003), no. 2, 186-204.

[21] Treves, F.: Basic linear partial differential equations. Pure and Applied Mathematics 62, Academic Press, New York-London, 1975.

Received August 16, 2012.

Jorge Clarke: Departamento de Matemática, Facultad de Ciencias, Universidad del Bio-Bio, Concepción, Chile; Laboratoire Paul Painlevé, Université de Lille 1, F-59655 Villeneuve d'Ascq, France; and $\mathrm{CI}^{2} \mathrm{MA}$, Departamento de Ingeniería Matemática, Universidad de Concepción, Concepción, Chile.

E-mail: jclarke@ubiobio.cl

Ciprian A. Tudor: Laboratoire Paul Painlevé, Université de Lille 1, F-59655 Villeneuve d'Ascq, France; and Academy of Economical Studies, Piata Romana nr. 6, sector 1, Bucharest, Romania.

E-mail: tudor@math.univ-lille1.fr

J. Clarke was partially supported by the MECESUP proyect UCO-0713 and the CONICYTECOS program C10E03. C. A. Tudor was supported by the CNCS grant PN-II-ID-PCCE-20112-0015 (Romania), and he is an associate member of the Samm group of the Université de Panthéon-Sorbonne Paris 1. The second author also acknowledges partial support from ANR "Masterie" BLANC 012103 (France). 